Thurax (1953), 8, 1.

\title{
A STUDY OF THE ORIGIN AND DEVELOPMENT OF LUNG CANCER
}

\author{
BY \\ C. RAEBURN AND H. SPENCER \\ From the Area Pathological Laboratory, Whipps Cross Hospital, London, and the Department of Pathology, \\ St. Thomas's Hospital Medical School, London
}

(RECEIVED FOR PUBLICATION JUNE 26, 1952)

During recent years increasing attention has been paid to the development and early stages of different forms of malignant neoplasms. The failure of the usual forms of treatment to arrest most well-established malignant tumours focused interest on the discovery of the earliest stages of development of such neoplasms, as it is only in the earliest phases that present methods of treatment offer any hope of effectual treatment. The study of the changes that lead on to established malignant tumour formation also provides possible clues to aetiological factors in some sites, and also enables some of the fundamental changes resulting in malignant tumour formation to be observed. It was with these objects in view that the present investigation was undertaken.

The terms "lung cancer" and "bronchial carcinoma" have been used synonymously, and it has been customary to consider that most lung cancers arise in the major bronchi near the hila of the lungs (Willis, 1948). Except for a few reported cases of small, peripherally situated tumours (Gray and Cordonnier, 1929; Stewart and Allison, 1943; James and Pagel, 1944; Petersen, Hunter, and Sneeden, 1949; Spain and Parsonnet, 1951; Prior and Jones, 1952) and one describing two minute growths in main bronchi (Aufses and Neuhof, 1952), very little attempt has been made to trace the origin of this very common tumour by looking for the earliest stages in its development. Because the larger bronchi are so frequently involved in the later spread of the disease it is usually assumed that most growths started in the main bronchi. That the epithelium and glands of the larger bronchi can and undoubtedly do provide the site of origin of primary carcinomata is certain, but the far greater surface area of epithelium provided by the peripheral bronchi and bronchiolar trees might well be expected to provide the site of origin for many more primary growths. Furthermore, the trachea and large bronchi undergo much of their development and differentiation in utero while the peripheral bronchi and bronchioles continue to develop during the first eight years of extra-uterine existence. Thus the peripheral air passages are the last parts of the air passages normally exposed to physiological developmental stimuli (Geschickter and Denison, 1934).

In view of the great frequency with which carcinoma of the lung is encountered at routine post-mortem examinations it might reasonably be expected that from time to time an unsuspected and early growth would be found somewhere in the lungs. Both of us for some years past have been in the habit of exploring the main bronchi and their immediate branches at all'post-mortem examinations in the hope that such an early growth would be discovered. So far only one such small carcinoma has been found in 750 adult post-mortem examinations.

The absence of such growths in the larger air passages prompted one of us to start sectioning the whole of both lungs with great care, and all suspicious nodules, particularly scars, were removed and sectioned as a routine. The results in the first 400 necropsies (Raeburn, 1951) yielded four small unsuspected carcinomata arising in the periphery of the lungs, mainly in association with scars. Since then our combined results of extensive searching in lungs obtained at routine post-mortem examinations, excluding infants, has revealed in a short period of a year a further six cases, including the one in the large bronchus mentioned above, which, when combined with three cases of small growths removed surgically, has provided 13 specimens of small and early growths (Cases 1-13), in which the mode and site of origin of the neoplastic process could be determined with reasonable accuracy.

In addition to the above tumours a further two cases (Cases 14 and 15) have been added. These, though very considerably larger, had arisen in sites other than main bronchi and appeared to have been intimately related to pre-existing chronic inflammatory lesions in the lung. 
The following is a brief description of the 15 cases forming the series.

\section{Summaries of 15 CASES}

CASE 1.-A man aged 68 died from renal failure due to bilateral hydronephrosis. A second primary papillary and transitional-celled bladder carcinoma had invaded and blocked both ureters. In addition there were considerable emphysematous changes in both lungs and slight roughening of the main right upper lobe bronchus, the mucous membrane of which was raised by several separate nodules up to $2.0 \mathrm{~mm}$. in diameter over a distance of $1.3 \mathrm{~cm}$. (Fig. 1).

CASE 2.-A woman aged 62 was known to have active pulmonary tuberculosis. Bronchoscopy was performed and a small nodular swelling approximately $3 \mathrm{~mm}$. in diameter was seen in the mucous membrane of the orifice of the main left upper lobe bronchus. The nodule and a strip of mucous membrane were removed. No obstruction of the bronchus was seen and all the visible growth was removed (Fig. 2).

CASE 3.-A man aged 58 gave a history of a chronic cough dating back 25 years and recent slight haemoptysis. Bronchoscopy showed a raised nodular growth in the right lower lobe main bronchus extending approximately $1.3 \mathrm{~cm}$. The growth was removed surgically together with four hilar glands, two of which appeared to contain growth. Section showed a squamous-celled carcinoma starting as an intra-epithelial cancer.

CASE 4.-A woman aged 50 had complained of slight haemoptysis. Bronchoscopy revealed a few small nodules, none of which exceeded 3 $\mathrm{mm}$. in diameter, in the mucous membrane of the right upper lobe main bronchus. One was excised for histological examination and showed an intraepithelial carcinoma.

CASE 5.-A woman aged 64 died of general peritonitis after a total gastrectomy for a columnar-celled adenocarcinoma. Post-mortem examination revealed an old, cavitated, fibrous scar $1.5 \mathrm{~cm}$. in diameter, which was considered to be tuberculous $ᄋ$ in nature, occupying the apex of the right $\gg$ lung. No growth was suspected on naked-eye examination and it was only found on microscopical examination (Figs. O 3 and 4).

CASE 6.-A woman aged 68 died of a massive pulmonary embolism. Postmortem examination showed a greyish. wedge-shaped scar beneath the pleura of the middle lobe of the right lung $0.3 \Phi$ $\mathrm{cm}$. in diameter. Fig. 5 shows the micro-? scopical appearance of the growth, whose $\frac{T}{0}$ existence had not been suspected previously. $\stackrel{\circ}{\circ}$

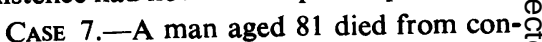
fluent bronchopneumonia. The apex of $\stackrel{\square}{\varrho}$ the left lung was fibrosed, and only when examined microscopically was a small oat-
FiG. 2.-Case 2: section of the main left upper lobar bronchus showing an intraepithelial carcinoma arising in metaplastic squamous bronchial epithe 
and round-celled carcinoma discovered.

CASE 8.-A man aged 73 died from myocardial infarction. $\mathrm{He}$ was also found to have syphilitic aortitis and a chronic gastric ulcer. The apex of the left lung was scarred. Only following microscopical examination was a very early carcinoma of a polygonal- and squamoidcelled type (Fig. 6) discovered.

CASE 9.-A man aged 59 died from generalized purulent bronchitis and emphysema. Post-mortem examination revealed a soft, greyish-white nodule $1.2 \mathrm{~cm}$. in diameter surrounding blood vessels in the upper lobe of the left lung. The nodule was close beneath the pleura in the paravertebral part of the lobe. A solitary metastasis was found in the left fifth rib. Microscopical examination revealed a mixed polygonal and squamous-celled growth arising in a preexisting scar (Fig. 7).

CASE 10.-A man of 76 died from confluent bronchopneumonia. There was a patch of pleural thickening on the surface of the right lower lobe $3 \mathrm{~cm}$. in extent and extending $0.6 \mathrm{~cm}$. into the subjacent lung. Microscopical examination revealed old scar tissue, reparative changes, and a polygonalcelled carcinoma developing in some places.

CASE 11.-A man aged $72^{\circ}$ died from rheumatic mitral stenosis and heart failure. A small greyish-white nodule $1.5 \mathrm{~cm}$. in diameter was found in the upper part of the lower lobe of the right lung. There was some scarring in the vicinity and an old calcified and caseous tuberculous focus in the apex of the right lung. Microscopical examination showed an adenocarcinoma arising in the edges of an old fibroanthracotic scar.

CASE 12.-A woman of 69 died of coronary artery occlusion. A greyish nodule, $0.2 \mathrm{~cm}$. in diameter $2 \mathrm{~cm}$. beneath the pleura was found in the lower lobe of the left lung. Microscopically it proved to be an oat- and roundcelled carcinoma with evidence of surrounding fibrosis due to previous damage of a lung.

CASE 13.-A man aged 64 died from a paraplegia complicated by acute pyelonephritis. Post-mortem examination showed that the upper lobe of the left lung was adherent to the chest wall over a circular area of approximately $2 \mathrm{~cm}$. Beneath the fibrous adhesions

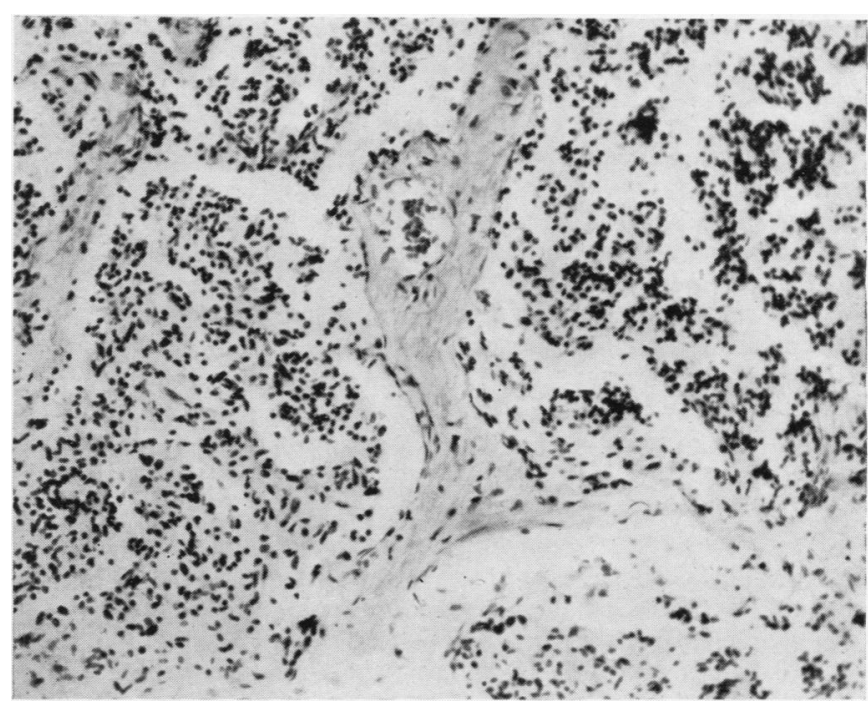

Fig. 4.-Case 5: a high-power view of the minute round- and oat-celled carcinoma seen in Fig. 3. The spindle shape, hyperchromatism, and arrangement of the cells differentiate this type of growth from a benign adenomatous tumour $(\times 140)$.

there was an indurated superficial scar in the lung, and deep to this was a greyish-white nodule $1 \mathrm{~cm}$. in diameter. The superficial scar and the deeper nodule together resembled a collar stud in shape. There was secondary growth in the lymph glands lying immediately external to the main lobar bronchus, and in a further gland behind the left clavicle. Examination of the spinal cord showed an area of softening at the level of D3-4, but no secondary growth to account for this 


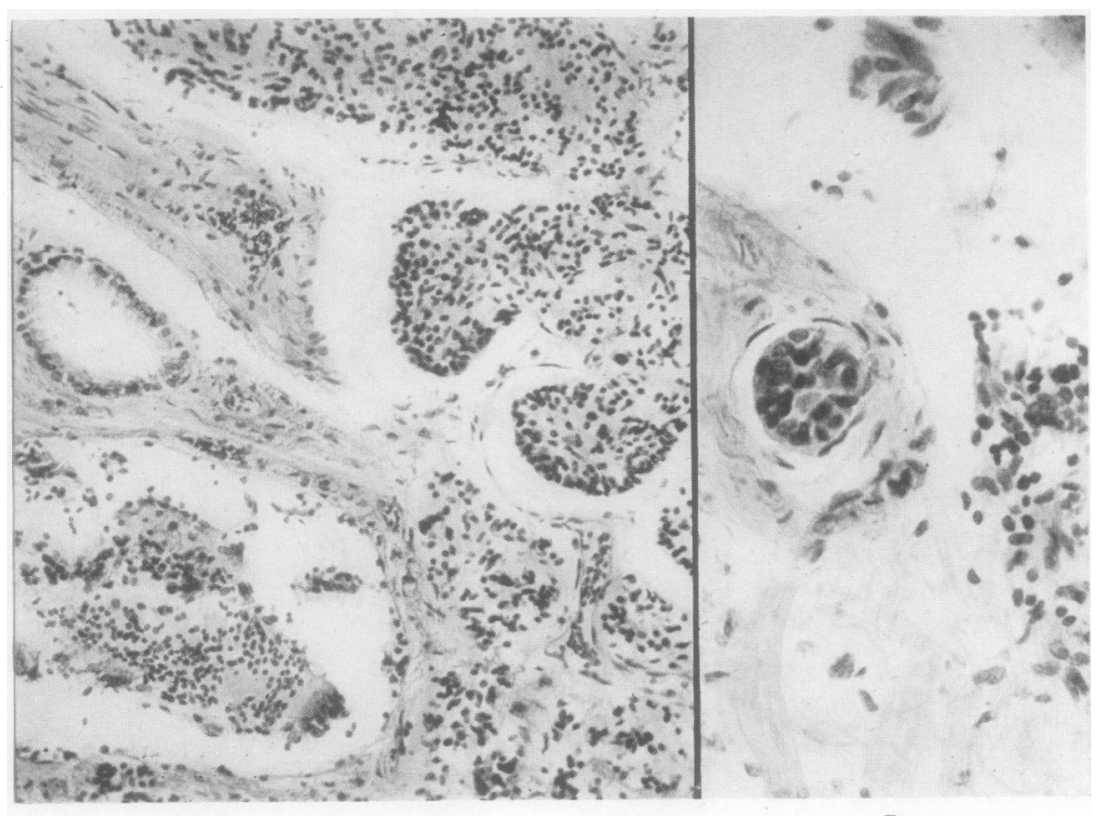

FIG. 5.-Case 6: A, a further example of a very small oat- and round-celled carcinoma arising in a scar $(\times 140) ; B$ shows the same growth within a lymphatic vessel $(\times 280)$

pale and more translucent. Microscopical examination showed a polygonal-celled carcinoma arising in and about a chronic fibroanthracotic scar.

Histological examination of the four growths found arising in the main lobar bronchi (Cases 1-4) showed that in every case they were squamous-celled carcinomata, and all four cases appeared to have started as intraepithelial cancer analogous to Bowen's intraepithelial skin cancer. The bronchial epithelium in some of the cases had undergone metaplastic squamous-celled change in sharply defined zones, and in these areas the epithelial cells were

was found in the cord. Microscopical examination showed an anaplastic predominantly round- and oatcelled carcinoma which had almost certainly arisen in connexion with the subpleural scar, and which had spread along the perivascular and peribronchial lymphatic to the central regional glands. From these glands, retrograde lymphatic spread into the submucous plexus of the lymphatic spaces of the upper lobe main bronchus had occurred, but there was no break of the surface mucosa that could be identified.

CASE 14.-A man aged 49 was found to have a shadow in the upper lobe of the right lung following mass radiological examination. He had no symptoms referable to the growth. The right lung was removed, and there was a deep puckered scar on the interlobar surface of the upper lobe. The middle lobe was adherent at this point. On cutting the lung there was a dense anthracotic scar extending inwards from the pleural surface surrounded by translucent growth approximately $2.5 \mathrm{~cm}$. in diameter. On microscopical examination it was found to be a mucus-secreting carcinoma arising in and about an old fibro-anthracotic lung scar. There was growth in one hilar gland with no detectable intermediately situated deposits.

CASE 15.-A man aged 50 complained of increasing dyspnoea, loss of weight, and a chronic cough. A shadow suggestive of a growth was seen in the lower lobe of the right lung. The right lung was removed, and on the posterior part of the lower lobe $5 \mathrm{~cm}$. above the diaphragm there was a dense scar extending into the lung from the pleural surface, the whole $3 \mathrm{~cm}$. in depth. The centre of this area was dark grey, the periphery hyperplastic, hyperchromatic, and some of the cells had enlarged, resembling the cells seen in Paget's disease of the breast, a condition probably analogous to that under discussion (Figs. 1 and 2). The ducts of the mucous glands had undergone squamous metaplastic change as in an intraepithelial ecto-cervical uterine cancer. The changes described above were multifocal, and it appeared as though a whole field of cells had undergone malignant change simultaneously. So far no other histological variety of lung cancer has been incontrovertibly proved to have arisen in a main bronchus. In the many other cases which we have examined, and where the larger bronchi were involved in growth, the appearances might equally well have been explained on a basis of secondary spread.

Although the aetiological factors concerned in most forms of human malignant disease are still unknown, the association of chronic irritation and inflammation as a precursor condition of carcinomatous growths in several situations is a wellestablished fact, and has been suggested as a cause for lung cancer (Wolf, 1895; Weller, 1929). The finding of unusual areas of hyperplasia and even malignant tumours associated with damage and fibrosis in the lungs might therefore be expected.

The investigation was also undertaken to determine whether there was any relationship between chronic inflammation in the lung and the subsequent 


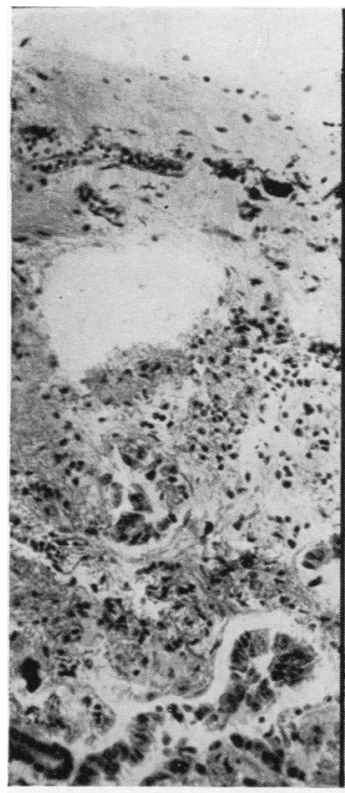

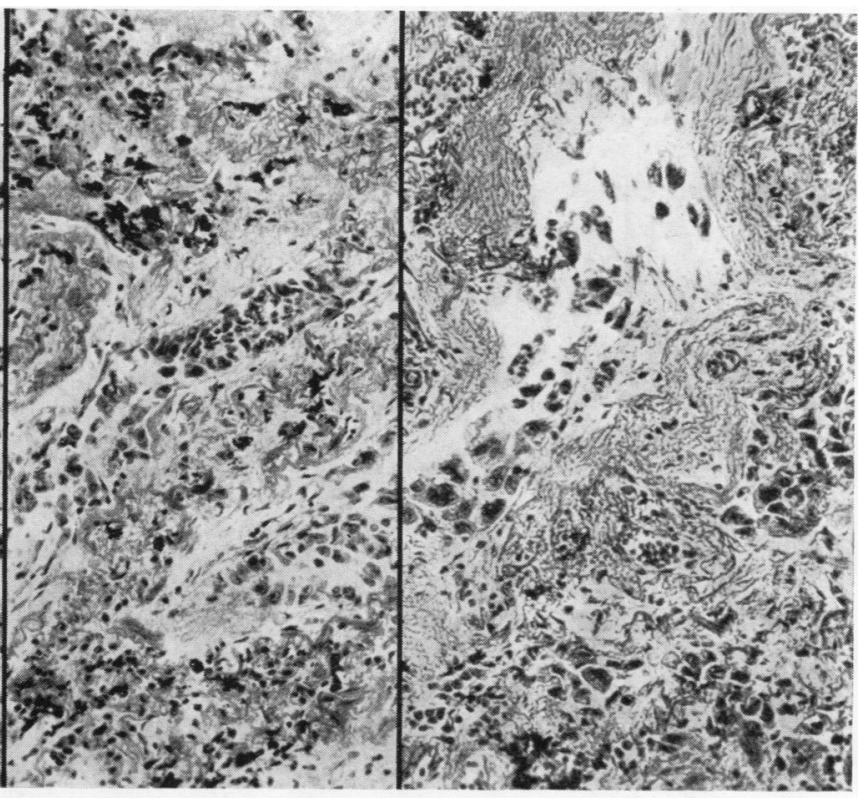

$\mathrm{C}$

Fig. 6.-Case 8: a composite photomicrograph of a scar cancer in the lung; A, normal bronchiolar proliferation at the edge of a small scar; B slightly deeper in the scar showing solid atypical bronchiolar outgrowths; C still deeper in the scar showing a polygonal-celled carcinoma (all $\times 140)$.

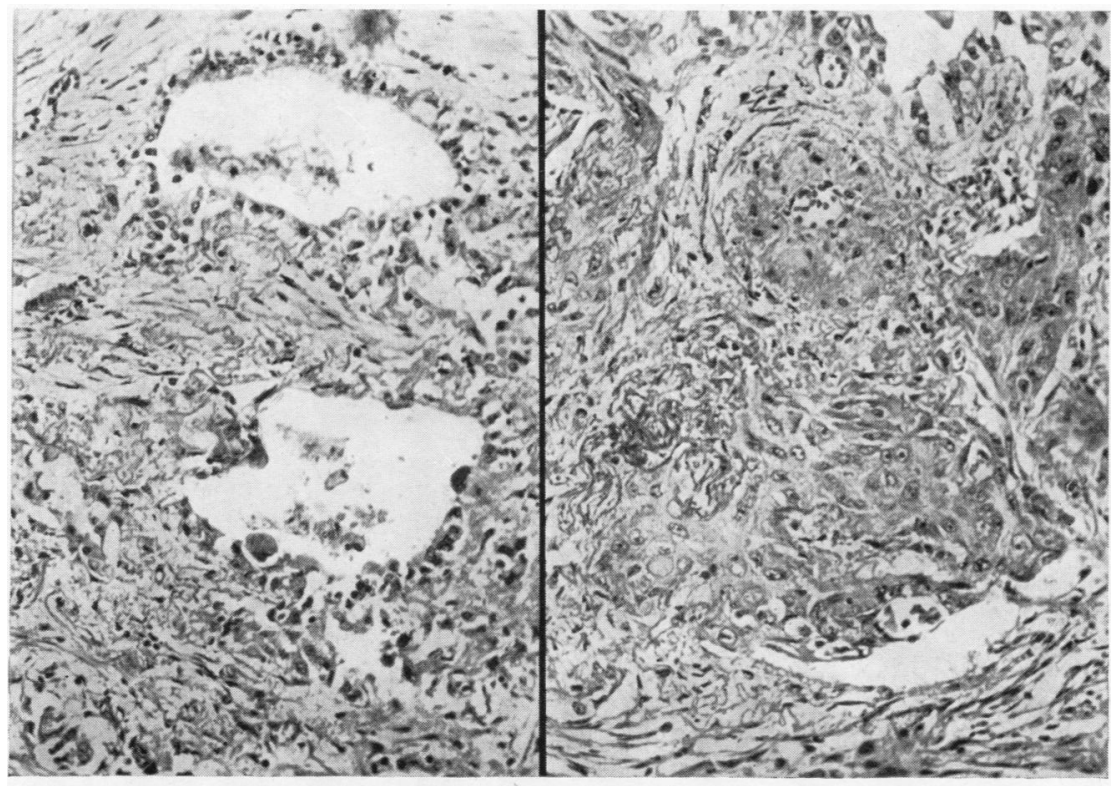

A

B

Fig. 7.-Case 9: A, bronchioles invading a lung scar (one of the bronchioles shows carcinomatous change); B, an adjacent microscopic field showing an area of squamoid carcinoma. An example of a scar cancer producing different histological varieties of growth in the same tumour $(\times 140)$. 
development of malignant change. As stated above, all scarred areas have been removed from both lungs at post-mortem examinations, including obvious tuberculous fibrocaseous and calcified nodules. Particular attention has lately been paid to lung tissue adjacent to scarred hilar lymph glands. Such material removed from 750 necropsies has enabled us to study the process of repair in a large number of scars in damaged lung, and has produced atypical areas of regenerative hyperplasia which we consider may be pre-cancerous, as well as 10 actual small or microscopical carcinomata.

The reaction of lung tissue to injury may be conveniently divided into the changes which occur in main bronchi and the changes which occur when bronchioles and alveoli are damaged. Macroscopic evidence of scarring in the larger bronchi is uncommon, and if the damage to the bronchial wall has involved the basement germinal layer of cells it frequently leads to destruction of the whole thickness of the bronchial wall. This is well demonstrated in suppurative bronchiectasis, where the bronchial wall may be destroyed and the surrounding lung becomes implicated in the destructive process. The wall of the resulting cavity is lined by fibrous tissue and may eventually become covered by squamous epithelium.

Lesser degrees of damage to the main bronchi, provided the basement layer of epithelial cells remains intact, is repaired by simple regeneration from the germinal layer of cells. The resulting

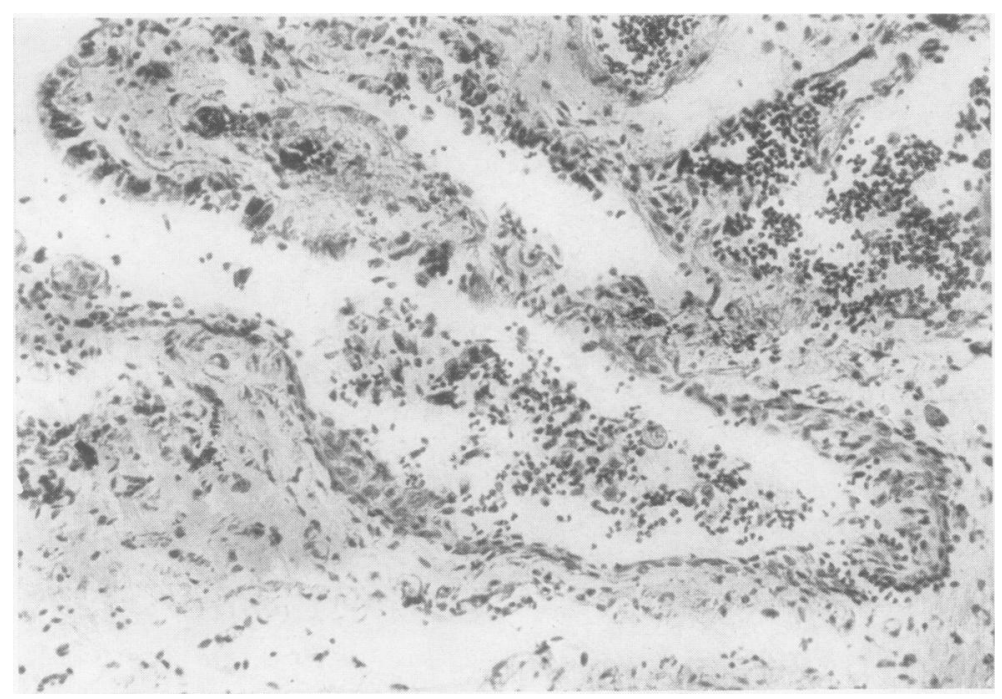

F1G. 8.-Case 6: a small bronchiole in an area of scarred iung adjacent to a microscopical oat- and round-celled carcinoma, showing squamous-celled metaplasia of the lining epithetium and bronchiolectasis $(\times 140)$ epithelium may undergo squamous metaplastic change, an increase in the number of mucus-secreting cells, or may return to its normal state.

Damage to the bronchioles and related lung alveoli evokes a different type of response. Minor degrees of bronchiolar and alveolar damage cause catarrhal inflammation in the bronchiolar epithelium and desquamation of the alveolar endothelial cells. Subsequently the bronchiolar epithelium may regenerate and spread down to line the damaged alveolar wall as a single layer of cubical epithelium. The bronchiolar epithelium if more seriously damaged may undergo squamous metaplasia like that in the larger air passages (Fig. 8).

Severe damage to bronchioles and lung parenchyma which leads to destruction of tissue stimulates a more complex reparative response. Montgomery (1944) studied the repair of wourds in the lung in animals, and showed that the bronchioles proliferated, and possibly because of the rhythmic respiratory movements and variation in intra-bronchiolar air pressure, the newly formed bronchioles grew into any available space and even split newly formed fibrous tissue. Similar changes explain the tortuous appearance of proliferating bronchioles seen in areas of damaged human lungs (Fig. 9). Subsequently portions of such newly formed bronchioles may become cut off from the parent air passage and then appear as isolated epithelial tubular or cystic structures embedded in scar tissue. In the majority of scars the process of repair is now completed, and the newly formed bronchioles often become cystic spaces lined by normal or inactive atrophic flattened epithelium. In some scars the bronchiolar proliferation may be so excessive that the appearances may come to resemble a fibroadenoma (Fig. 10). It is this latter variety of repair process which we have found may proceed in some cases to develop malignant change.

By carefully examining large numbers of scars it has been possible to find examples where the newly formed bronchioles have continued to undergo hyperplastic change and the epithelial cells filled up the lumen to form solid cords of cel!s not unlike the intraductular changes that may be found in 
chronic mastitis (Fig. 11). Also examples where there has been an outwardly directed proliferation of bronchiolar cells into the adjacent lung have been seen (Fig. 12). These collections of hyperplastic cells frequently bear a superficial resemblance to the carcinoid type of bronchial adenoma.

That only certain scars should proceed to develop atypical epithelial changes is not surprising. Inherited susceptibility to malignant change and the nature of the agent responsible for the damage may be among the more important factors determining whether malignant change may supervene. An analogous condition is found in the skin where scars following trauma seldom undergo squamous-celled carcinomatous change, but scars resulting from lupus vulgaris and $x$-ray radiation are notoriously liable to develop such malignant change after a prolonged interval.

Among the small neoplasms so far discovered in the periphery of the lungs were a group of four tumours which all bore a close resemblance to each other (Cases 5, 6, 7, and 12). They were all found close beneath the pleural surface of the lungs, and all were associated with evidence of chronic scarring of the lung and bronchiolectasis. In Case 6 the growth appeared to be arising multicentrically and showed indisputable evidence of emboli of cells in lymphatic endotheliallined spaces distant from the main body of the tumour. The cells composing the tumours were round, oat- and spindle-shaped and showed no mitotic figures but considerable hyperchromatism and variability in cell size and shape. Despite some

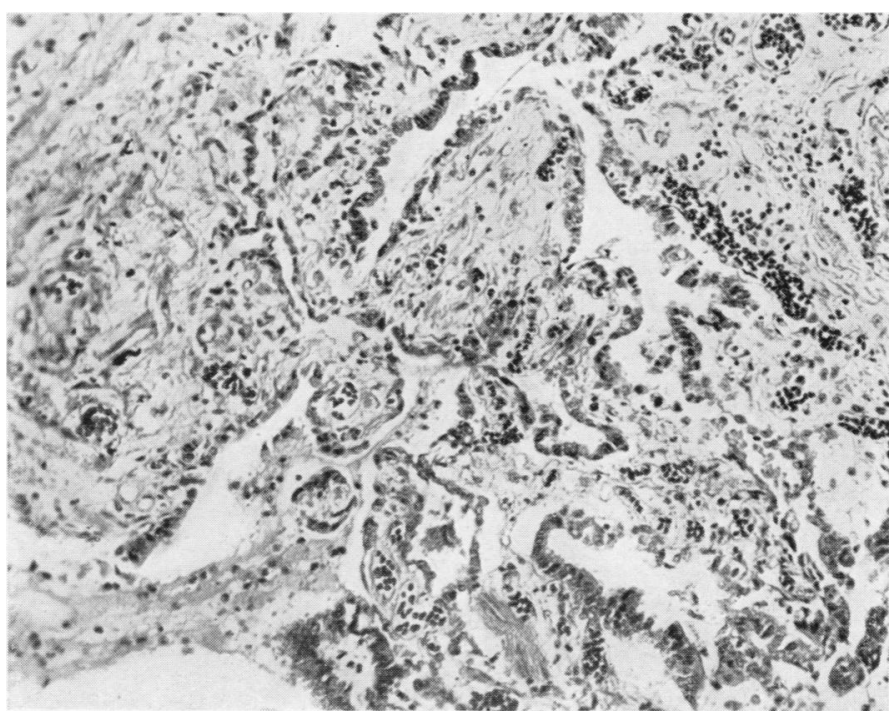

FIG. 9.-Section showing innocent bronchiolar proliferation in an area of scarring in the lung $(\times 140)$.

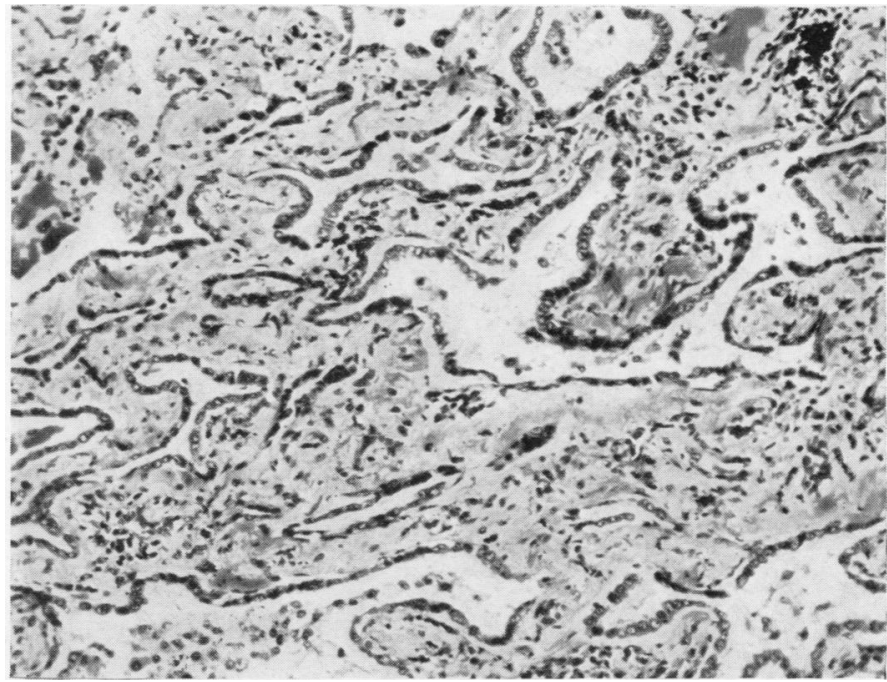

FIG. 10.-Case 10: a sub-pleural scar showing innocent bronchiolar proliferation resembling a fibroadenoma. The same scar also contained an undifferentiated polygonal-celled carcinoma in an adjacent microscopic field $(\times 140)$. cytological resemblance to the carcinoid typz of bronchial adenoma, the diffuse infiltrating nature of the growths, variability of cell pattern, and in at least two cases the presence of lymphatic permeation have led us to regard these four tumours as malignant and related cytologically to the oat and small round-celled carcinomata of the lung (Figs. 3, 4, and 5). The absence of mitoses and necrosis may indicate that these tumours are initially of a low-grade degree of malignancy and only represent a further stage in the evolution of the reparative hyperplastic changes already noted in Fig. 11.

The remaining four small and two larger peripheral growths (Cases 8, 9, 10, 11, and Cases 14 and 15) have been representative of all other common histological varieties of lung cancer. The outstanding feature has been the bronchiolar proliferation within scar tissue progressirg to malignant change (Fig. 6). Although most of the growths showed a tubular structure, squamous-celled areas were also found (Fig. 7). During the investigation 


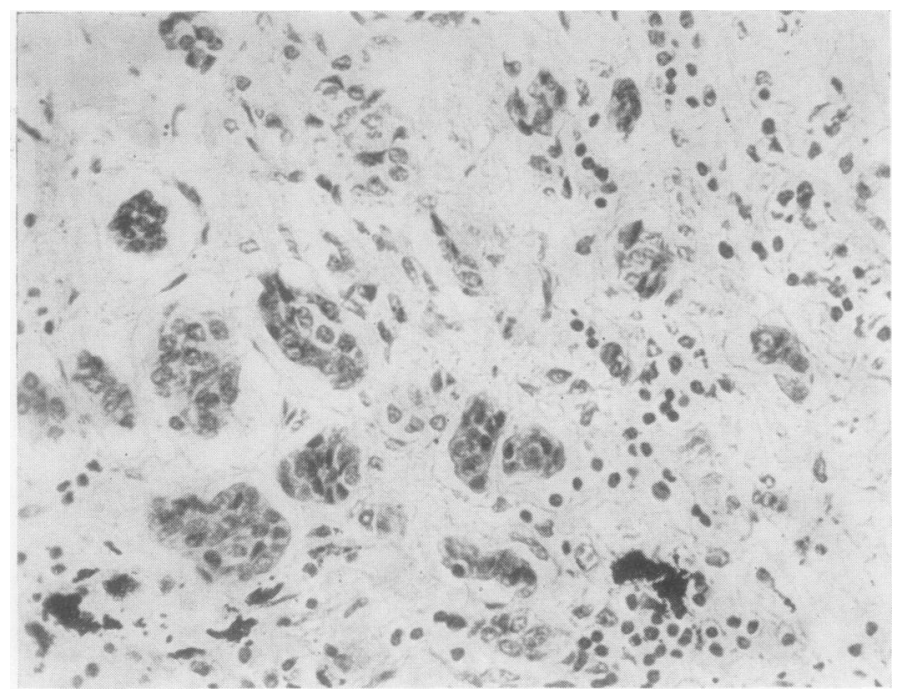

FIG. 11.-Bronchiolar proliferation in a scar in lung. The bronchiolar epithelium is forming solid nests of cells. The change was localized and was due to innocent regenerative hyperplasia $(\times 280)$.

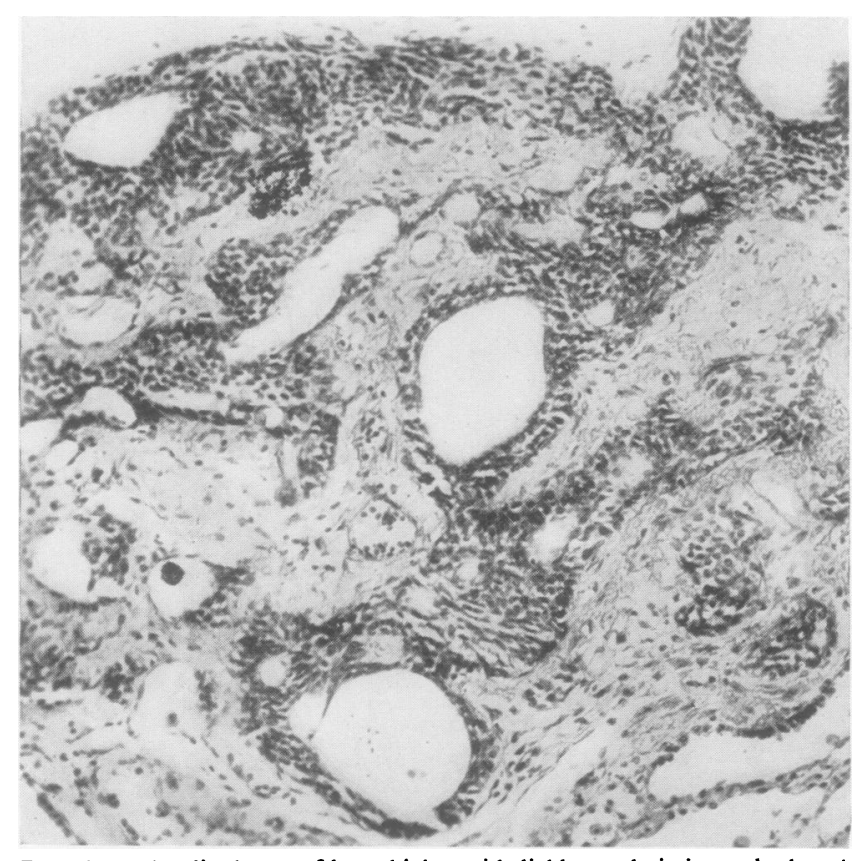

FIG. 12.-A localized area of bronchiolar epithelial hyperplasia in a sub-pleural scar, but showing no evidence of true malignant change $(\times 140)$.

many lung scars have bsen found which have shown all degrees of bronchiolar proliferation, many resembling fibroadenomata, and great difficulty has been experienced in determining the borderline between innocent reparative proliferation and true malignant change. Only cases which have shown obvious malignant change have been included in this series. Unfortunately, post-mortem examinations on patients dying from established lung cancer rarely afford much useful information, as the size of the growths precludes any attempt to determine their point of origin.

The rapidity with which many lung carcinomata metastasize and the occasional difficulty which a pathologist may experience in finding the site of a small primary growth are two features of the condition, both of which are well recognized. One of the minute scar cancers included in this series (Case 9), a mixed squamous-celled and adenocarcinoma (Fig. 7), had already metastasized to a rib without any evidence of distant lymphatic permeation, and Case 13 showed widespread lymphatic permeation which had reached the hilar lymph glands and the lymphatic channels in the lobar bronchial wall.

A further growth, while still fairly small and confined to the periphery of the lung, had already spread by the lymphatic channels to a central hilar gland which was adherent to the outer wall of the main upper lobe bronchus (Case 14). The possibility of further spread of growth from such a central lymph gland into the wall of the adjacent main lobar bronchus could lead to the mistaken impression that the growth had originated in the large bronchus.

\section{DISCUSSION}

Hitherto it has been customary to regard most lung carcinomata as having arisen from main bronchi and their immediate divisions, largely because the main bronchi so frequently become involved in a mass of growth. Although main bronchi undoubtedly can be the seat of origin of primary lung carcinomata, where we have been able to procure very
small early growths before spread has occurred the growths have in every case been of the intra-epithelial variety analogous to Bowen's disease of the skin, intra-epithelial cancer of the 
cervix uteri and oesophagus, ard Paget's intraepithelial cancer of the breast. Such carcinomata undoubtedly can metastasize and then usually behave as a fairly rapidly spreading variety of squamous-celled carcinoma. Apart from this type of growth, which starts ab initio as multiple areas of epithelial hyperplasia, all the other small growths which have been obtained from lungs show unmistakable evidence of having arisen in and about damaged lung tissue. In these cases the whole process leading up to malignant tumour formation appears to pursue a different course from those arising in the main bronchi. To appreciate the changes it is necessary to study the response of bronchiolar and alveolar tissue to damage. The bronchioles appear to play an important part in the healing process both by downgrowth of bronchiolar epithelium to line the partly damaged alveolar walls and by the formation of new bronchioles resembling the formation of new bile ducts following damage to the liver.

In most cases in which lung is damaged the bronchiolar changes described above take place, but after an initial period of hyperplasia the newly formed epithelium becomes flattened and atrophic and all evidence of active growth ceases. In a few cases, however, possibly over a long period, regenerative hyperplasia continues and results in atypical areas of epithelial proliferation. The bronchiolar epithelium becomes heaped up both within the lumen of damaged alveoli and new bronchioles, and the resulting condition resembles an innocent tumour. The border-line between innocency and malignancy, as in so many other situations, is very indeterminate, and other cases show unmistakable features of being true carcinomata.

Among the group of peripherally arising tumours, four showed a very close resemblance to each other and to other similar tumours recently reviewed by Prior and Jones (1952). Although for the reasons already stated, namely local infiltration, lymphatic permeation, and cytological structure, we have included these growths as early carcinomata and not among the adenomata, our cases in common with other similar cases previously reported in the literature show certain unusual features. Of the 19 cases collected by the authors we have cited, 14 occurred in women and only five in men, a reversal of the usual sex incidence found in lung cancer. This unusual sex incidence has been found also in our four cases, with three female and one male case. The sub-pleural localization, and the association of these tumours with lung fibrosis and bronchiolectasis also noted by the same authors, was also common to our four cases. As in the case reported by Gray and Cordonnier, one of our tumours (Case 6) was multicentric in its points of origin. Unfortunately the future course of the patients found harbouring these growths was impossible to assess, although the patient who was reported by Stewart and Allison in 1943 as showing a similar tumour in a brochiectatic lung has remained alive and well.

Future study of this distinctive group of tumours may show that in fact they belong to a group of relatively benign adenomatous tumours or hyperplasias and should not be included among the malignant lung tumours.

Of the remaining seven undoubted peripherally situated scar cancers, all were found in males and three were almost entirely of an adenocarcinomatous pattern. This conforms to the almost universal finding of male preponderance in true lung cancer and to the observed greater frequency of origin of adenocarcinomata in the periphery of the lung. The growth found in Case 8 and shown in Fig. 7 is very similar to that in the two cases described by James and Pagel (1944), and the description of their cases leaves little doubt that their two cases were but further examples of malignant change supervening in lung scar tissue at the apices of lungs.

This latter group of tumours showed local infiltration, lymphatic permeation, and even bloodborne metastases at a very early stage in their development.

The relationship of previous lung damage to primary malignant disease of the lung has been noted before, but the present tendency is to regard most lung tumours as having arisen from the lobar bronchial epithelium or glands. From our experience it would appear possible that some growths start in the lung substance from bronchiolar tissue in areas of chronic fibrosis and thence spread to the regional hilar glands. From the regional hilar glands subsequent backspread into the lymphatic channels in the adjacent lobar bronchial wall may then occur.

The almost universal finding of at least one or two scarred areas in the lung substance at postmortem examination contrasts with the rarity of naked-eye and even microscopical evidence of chronic damage to the main bronchi and their surface epithelium. This fact, combined with the known terdency in many situations for chronic inflammation and fibrosis to precede malignant change, agrees more closely with our observed findings of malignant growths and atypical areas of epithelial hyperplasia arising in fibrotic and chronically inflamed lung tissue rather than in the 
main bronchi, which seldom show evidence of such damage.

The possibility that the scar tissue around the growths might have resulted from host reaction to the presence of tumour cells, rather than being a causative factor in the origin of the carcinomata, has been considered. Careful comparison of the structure of scar cancer and simple lung scars, and the ability to demonstrate all stages leading to malignant change in the latter, has convinced us, apart from other reasons, that the scarring antedated the appearance of malignant changes.

\section{SUMMARY}

Fifteen lung cancers, including 13 small or microscopical growths, have been collected and examined in an attempt to trace the origins of this common tumour. Four of the neoplasms, all arising in main lobar bronchi, were found to start as intra-epithelial carcinomata. The histology of these neoplasms resembled the Bowen's type of skin cancer and intra-epithelial carcinomata arising in other sites.

Of the remaining 11 cases, all were found in association with scars situated near the periphery of the lungs. Four of the peripheral growths showed features resembling the carcinoid type of bronchial adenomata, but provisionally have been regarded as carcinomata of the oat-celled type.

Several stages could be traced between simple bronchiolar regeneration in and around lung scars and fully developed carcinomata. Many of the latter were still microscopical in size.

It is suggested that many so-called "central" lung cancers do not arise in the lobar bronchial epithelium, but involve these structures as a result of secondary spread from smaller and distant growths near the periphery of the lung.

We wish to thank Professor W. G. Barnard for his interest and advice in the preparation of this paper and Mr. A. E. Clarke for the photomicrographs.

\section{REFERENCES}

Aufses, A. H., and Neuhof, H. (1952). J. thorac. Surg., 23, 219.

Geschickter, C. F., and Denison, R. (1934). Amer. J. Cancer, 22, 854 Gray, S. H., and Cordonnier, J. (1929). Arch. Surg., Chicago, 19. ray, S. H.

James, I., and Pagel, W. (1944). Brit. J. Surg., 32, 85

Montgomery. G. L. (1944). lbid., 31, 292.

Petersen, A. B., Hunter, W. C., and Sneeden, V. D. (1949). Cancer, 2,991 .

Prior, J. T., and Jones, D. B. (1952). J. thorac. Surg., 23, 224

Raeburn, C. (1951). Lancet, 2, 474 .

Raeburn, D. M., and Parsonnet, V. (1951). Cancer, 4, 277.

Stewart, M. J., and Allison, P. R. (1943). J. Path. Bact., 55, 105.

Weller, C. V. (1929). Arch. Path., Chicago, 7, 478.

Willis, R. A. (1948). The Pathology of Tumours, p. 359. London

Wolf, K. (1895). Fortschr. Med., 13, 725 . 